\title{
Analysis of thermal performance of geophonic down-hole measuring tools; A numerical and experimental investigation
}

\author{
Rohitha Weerasinghe \\ Department of Engineering Design and Mathematics, Faculty of Environment and \\ Technology, University of the West of England,Coldharbour Lane, Frenchay,Bristol BS16 \\ $1 Q Y, U K$ \\ Thomas Hughes \\ Department of Engineering Design and Mathematics, Faculty of Environment and \\ Technology, University of the West of England,Coldharbour Lane, Frenchay,Bristol BS16 \\ $1 Q Y, U K$
}

\begin{abstract}
Downhole tools encounter harsh environmental conditions due to pressure and and elevated temperatures. Use of Peltier cooling in down-hole seismic tooling has been restricted by the performance of such devices at elevated temperatures. Present paper analyses the performance of Peltier cooling in temperatures suited for down-hole measuring equipment using measurements, predicted manufacturer data and computational fluid dynamic analysis. A critical analysis of Peltier performance prediction techniques is presented with measurements. Validity of the extrapolation of thermoelectric cooling performance at elevated temperatures has been tested using computational models for thermoelectric cooling device. This method has been used to model cooling characteristics of a prototype downhole tool and the computational technique used in has proven valid. Further, an CFD analysis of the performance of two heat sink metals has been presented. The experimental and modelling exercise was targeted at achieving cooling performance that would enable the tool withstand temperatures near $200{ }^{\circ} \mathrm{C}$
\end{abstract}

Keywords:

Geophonic resonance, thermoelectric cooling, Peltier cooling, down-hole tools, thermal boundary layer, thermoelectric refrigeration, CFD, thermal 
management

\section{Introduction}

Geophonic Seismic resonance measuring tools are sensitive devices. The accuracy of the measurements, and correct operation is ensured only if they are operating below permissible temperatures. The phenomenon that a voltage is generated in a conductor or semi conductor subject to a temperature gradient was discovered around 1800 and is known as the Seebeck effect. The inverse process where heat is pumped across a conductor or a semi conductor due to a voltage difference is known as the Peltier effect. The current uses of the Seebeck effect are mainly for thermoelectric heat recovery. Nevertheless, thermoelectric cooling is used in applications where space is limited and where conventional refrigerants cannot be used, for example in computers and small machinery.

The traditional operating range for electronics [9] has been $-65{ }^{\circ} \mathrm{C}$ to $+125{ }^{\circ} \mathrm{C}$. Extreme temperature electronics [18] cover the temperature range of absolute zero to temperatures as high as $+400{ }^{\circ} \mathrm{C}$. However, total circuits cannot withstand these limits. The oldest, and currently largest, user of hightemperature electronics (temperatures exceeding $150^{\circ} \mathrm{C}$ ) is the downhole oil and gas industry [26]. In downhole application, the operating temperature is a function of the underground depth of the well. Worldwide, the typical geothermal gradient is $25^{\circ} \mathrm{C} / \mathrm{km}$ depth, or even higher[20].

During deep oil and gas well logging, the electronic modules inside the logging tool have to endure wellbore temperature of $200^{\circ}$ for hours. However, typically allowable operation temperature of most commercially available electronics is no more than $120^{\circ}[5,34]$. Therefore, not all the downhole electronic components can withstand severe ambient temperature exceeding $200^{\circ}$ due to a decay of reliability or even complete failure. The effects of harsh thermal environemnts have been studied by $[5,34]$. One of the most challenging problem is the decayed reliability of heat-sensitive electronic module at surrounding temperature exceeding $200^{\circ}$. To overcome this challenge, an alternative option is the use of electronic modules that are custom made for survivability at high temperatures. Despite the commercial availability of high temperature compatible electronics which can ensure the reliability of electronic at high ambient temperature, the available high-temperature electronic components can be very expensive. In this case, thermal man- 
agement is essential to protect heat-sensitive electronics from harsh thermal environments [14].

This means that key device thermal characteristics are crucial for successful, high-performance operation at elevated temperatures [32]. One of the most important and well-known challenges is posed by increased substrate leakage current. Some others are decreased carrier mobility, variation in device parameters, such as threshold voltage, gain, and saturation voltage, increased electromigration of metal interconnects, and decreased dielectric breakdown strength. Although standard silicon can operate well beyond the military requirement of $125^{\circ} \mathrm{C}$, leakage in standard silicon processes doubles for every $10 \mathrm{~K}$ increase, making it unacceptable for many precision applications.

Trench isolation, silicon-on-insulator (SOI), and other variations on the standard silicon process greatly decrease leakage and enable high-performance operation to well above $200{ }^{\circ} \mathrm{C}$. Wide-band-gap materials, such as silicon carbide $(\mathrm{SiC})$, raise the bar even higher; silicon carbide ICs have operated at up to $600{ }^{\circ} \mathrm{C}$ in laboratory investigations. However, $\mathrm{SiC}$ is an emerging process technology, and, currently, only simple devices such as power switches are commercially available [32].

Cooling of downhole electronics can be divided into active cooling and passive cooling. Active cooling methods are far superior in performance than passive cooling. However, they cannot be readily used in measuring tools as they often come with cooling liquid and moving parts which makes it unsuitable to use in the downhole measuring environments. active cooling methods, such as vapor compression cooling and refrigerator circulation cooling, are undesirable for wireline logging. Therefore, for wire-line logging, passive downhole electronics cooling techniques are wildly used, including thermal insulation [23], thermo-electric cooling [8, 22], and phase change material (PCM) for heat storage [31, 30]. A common technique in passive cooling, finned heat sinks are not an alternative because of narrow air gap inside the wireline logging tool.

Thermoelectric cooling has been around since the early 1950s and extensive research has taken place in aid of cooling at room temperature and around. Most these applications happen around ambient temperature [17]. The main disadvantages of thermoelectric cooling are the high cost and low energy efficiency, which has restricted its application to cases where system cost and energy efficiency are less important than energy availability, system reliability and quiet operation environment. The application of thermoelec- 
tric cooling in the present work is important because other methods of cooling have not given the desired qualities that are necessary for the downhole environment. Other viable alternative that can be used instead of or jointly with Peltier devices is phase change materials [28, 14].

Despite thermoelectric cooling effect was discovered in the 19th century, it hadnt come to rapid development until 1950s when the basic science of thermoelectric materials became well established [35]. Manufacturers of Peltier conductors have tested their devices at room temperature and within a band of $30 \mathrm{~K}$ either side of that. Seismic down-hole measuring devices operate under harsh environmental conditions, a few kilometres below ground at temperatures that are $200 \mathrm{~K}$ above standard room temperature. These tools contain sensitive electronic circuitry and the performance of the devices depend on whether these devices can be kept within the operating temperatures of the electronics. Due to the harsh conditions and heat accumulation it is important that the heat is being pumped to cool the electronics. A lot of studies have taken place in thermoelectric heat recovery at elevated temperatures and representative data are available[2]. However, the availability of representative data in cooling at these temperatures are rare. As a representative technique, the properties are being extrapolated from room temperature results [12]. Thermoelectric cooling in seismic measuring tools is a novel technology. The performance of such devices has been hampered by the relatively low overall coefficient of performance. However, this low COP has been resulted mostly by the losses in the thermal passage of such devices. Thermal analysis has shown that the performance can be enhanced by better insulation the device and carefully arranging the thermal paths. Heat transfer modelling has helped improve this aspect of the measuring tools.

Thermal analysis of Peltier performance can be used to analyse the overall device performance by identifying the heat transfer patterns, paths and the actual cooling performance of the Peltier device [30]. Present study looks at the performance of the Peltier devices in cooling at elevated temperatures and the validity of heat transfer models used to predict the properties[11]. The cooling performance of the exiting devices are such that a $10 \mathrm{~K}$ improvement in the maximum temperature means a significant achievement. 


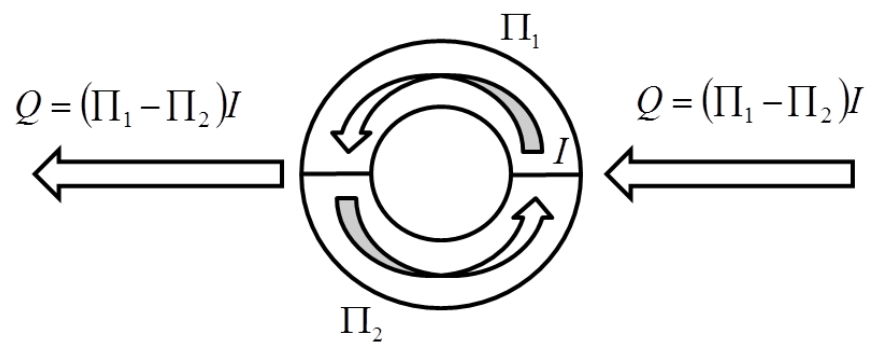

Figure 1: Figure shows how Peltier effect takes place at junctions of two materials

\section{Peltier Cooling}

The amount of heating or cooling obtained using a thermoelectric device depends on its thermal efficiency. The maximum efficiency of a thermoelectric device for both power generation and cooling is determined by [29]

$$
Z T=\frac{S^{2} \sigma}{\kappa} T
$$

Here, $Z T$ is the dimensionless quantity figure of merit, $S$ the Seebeck coefficient, $\sigma$ electrical conductivity and $\kappa$ the thermal conductivity. $\kappa$ is composed of lattice thermal conductivity $\kappa_{p}$ and electronic thermal conductivity $\kappa_{e}$. The Seebeck coefficient is the voltage generated per degree of temperature difference over a material. $S^{2} \sigma$ actually represents the power factor, a large power factor means that electrons are efficient in heat-electricity conversion whilst a small thermal conductivity is required to maintain a low temperature gradient, thus minimising losses [24]. Thermoelectric materials have been studied recently in the forms of bulk thermoelectric materials, individual nanostructures, bulk nanostructures, and interfaces in bulk thermoelectric materials. Peltier heating and cooling happens when two materials with two different Peltier coefficients are joined together. Due to the imbalance of the Peltier heat flow in and out of junction. The cooling or heating, $Q$ happening at the junction is equal to

$$
\dot{Q}=\left(\Pi_{2}-\Pi_{1}\right) I
$$

where $I$ is the electrical current, $\Pi$ Peltier coefficient with subscripts 1 and 2 representing the two materials. 


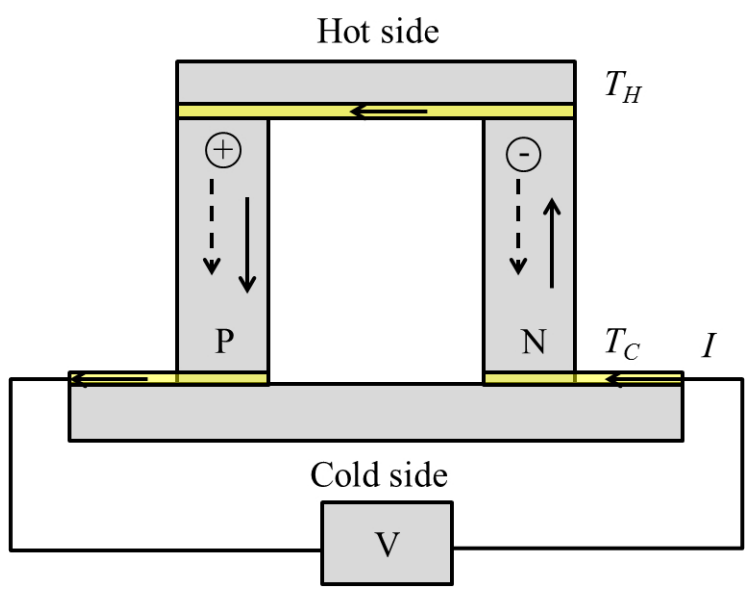

Figure 2: Thermoelectric cooling with one $p$-n pair

Thermoelectric devices consist of many pairs of p-type and n-type semiconductor pellets connected electrically in series and thermally in parallel. The temperature distribution can be obtained by solving for one dimensional transport along the pellet axis[7]. Figure 2 shows how the semiconductors are paired between an upper temperature of $T_{H}$ and a lower temperature of $T_{C}$. The heat absorption rate by the lower boundary is given by the equation $[35]$

$$
\dot{Q}_{C, p}=S_{p} I T_{C}-\frac{I^{2} \rho_{p} L_{p}}{2 A_{p}}-\frac{\kappa_{p} A_{p}\left(T_{H}-T_{C}\right)}{L_{p}}
$$

where $\rho=1 / \sigma$ is the electrical resistivity, $A$, cross sectional area of the leg, and, $L$, the length of leg. Similarly, the rate of heat pumped to the upper boundary is given by

$$
\dot{Q}_{H, p}=S_{p} I T_{H}+\frac{I^{2} \rho_{p} L_{p}}{2 A_{p}}-\frac{\kappa_{p} A_{p}\left(T_{H}-T_{C}\right)}{L_{p}}
$$

The difference between these two is the work rate, which is given by

$$
\dot{W}=S_{p} I\left(T_{H}-T_{C}\right)+\frac{I^{2} \rho_{p} L_{p}}{A_{p}}
$$

For a cooling device, the resistance $R$ and thermal conductivity $\kappa$ can be 
defined as

$$
R=\frac{L_{p} \rho_{p}}{A_{p}}+\frac{L_{n} \rho_{n}}{A_{n}}
$$

and

$$
\kappa=\frac{\kappa_{p} A_{p}}{L_{p}}+\frac{\kappa_{n} A_{n}}{L_{n}}
$$

Here, $L$ stands for length of the leg, $\rho$ the density, and $A$ cross sectional area. Subscripts $p$ and $n$ represent $\mathrm{p}$ and $\mathrm{n}$ type poles. A Peltier device is made of a number of thermocouples instead of a single module. The heat fluxes for $N$ thermocouples become

$$
\begin{aligned}
& \dot{Q}_{C, p}=N\left[S_{p} I T_{C}-\frac{I^{2} \rho_{p} L_{p}}{2 A_{p}}-\frac{\kappa_{p} A_{p}\left(T_{H}-T_{C}\right)}{L_{p}}\right], \\
& \dot{Q}_{H, p}=N\left[S_{p} I T_{H}+\frac{I^{2} \rho_{p} L_{p}}{2 A_{p}}-\frac{\kappa_{p} A_{p}\left(T_{H}-T_{C}\right)}{L_{p}}\right]
\end{aligned}
$$

and the work rate

$$
\dot{W}_{T E M}=N\left[S_{p} I\left(T_{H}-T_{C}\right)+\frac{I^{2} \rho_{p} L_{p}}{A_{p}}\right]
$$

The coefficient of performance (COP) of cooling can be stated by [27]

$$
\mathrm{COP}=\frac{S_{p} I T_{C}-\frac{I^{2} \rho_{p} L_{p}}{2 A_{p}}-\frac{\kappa_{p} A_{p}\left(T_{H}-T_{C}\right)}{L_{p}}}{S_{p} I\left(T_{H}-T_{C}\right)+\frac{I^{2} \rho_{p} L_{p}}{A_{p}}}
$$

Here, $S$ refers to the Seebeck coefficient and the subscripts $p$ and $n$ refer to $\mathrm{p}$ and $\mathrm{n}$ type semi-conductors, respectively. $T$ denotes temperature where subscripts $H$ and $C$ denote hot and cold sides.

\section{Modelling of Thermoelectric Cooling}

The heat transfer equation for thermoelectric cooling can be written as follows following equation (8);

$$
\dot{Q}_{C}=2 N\left[S I T_{C}-\left(\frac{I^{2} \rho}{2 G}\right)-\kappa\left(T_{H}-T_{C}\right) G\right]
$$


where $G$ is the area factor (area/length), and the maximum temperature difference is given by

$$
\left(T_{H}-T_{C}\right)_{\max }=T_{H}-\left[\frac{\sqrt{1+2 Z T_{H}}-1}{Z}\right]
$$

and the electrical voltage across the module

$$
V=2 N\left[\frac{I \rho}{G}+S\left(T_{H}-T_{C}\right)\right]
$$

and

$$
I_{\max }=\frac{\kappa G}{S}\left[\sqrt{1+2 Z T_{H}}-1\right]
$$

These equations are simplified in the numerical simulation engine, and the heat fluxes are expressed as

$$
\dot{Q}_{C}=(S I+K) T_{C}-K T_{H}-\frac{1}{2} I^{2} R
$$

and

$$
\dot{Q}_{H}=K T_{C}-(S I-K) T_{H}-\frac{1}{2} I^{2} R
$$

and

$$
V=S\left(T_{H}-T_{C}\right)+I R
$$

The module thermal conductivity $K$ is taken as $2 \kappa N G$. Based on the above, the Seebeck coefficient $S$ and the module thermal conductivity $K$ can be written as

$$
S=\sqrt{\frac{2 R Q_{\max }}{T_{H}^{2}}}
$$

and

$$
K=\frac{S^{2}\left(T_{H}-\Delta T_{\max }\right)^{2}}{2 R \Delta T_{\max }}
$$

Values of $K$ are available from manufacturers based on near room temperature measurements performed at controlled conditions. Such manufacturer data are tabulated in terms of Seebeck coefficient $S$ and the module thermal conductivity $K$. The performance of the thermoelectric device is described 
using the main parameters coefficient of performance $(Z)$ and the dimensionless figure of merit where $R_{L}$ is the load resistance. The overall efficiency of the unit is given by the coefficient of performance [6]

$$
z=\frac{\dot{Q}_{C}}{\dot{Q}_{H}}
$$

and, the figure of merit

$$
Z T=\frac{S R_{T}}{K R}
$$

These values are then used to fit a linear equation for the Seebeck coefficient.

\section{Measurement of thermoelectric cooling performance}

In order to model the thermoelectric cooling performance using mathematical modelling, in this case, the CFD programme Star CCM+, it is necessary to find the temperature dependent properties [21]. These properties have been tabulated by the manufacturer for low temperatures[10]. The temperature performance has been predicted using derived linear equations [13]. Table 1 shows the values available from manufacturer data. These

Table 1: Manufacturer data for thermoelectric module

\begin{tabular}{lll}
\hline$T_{H}\left({ }^{\circ} \mathrm{C}\right)$ & 25 & 50 \\
\hline$T_{H}(\mathrm{~K})$ & 298.15 & 323.15 \\
$Q_{\max }(\mathrm{W})$ & 126 & 138 \\
$\Delta T_{\max }(\mathrm{K})$ & 67 & 76 \\
$I_{\max }(\mathrm{A})$ & 14.6 & 14.5 \\
$V_{\max }$ & 14.4 & 16.2 \\
$R(\Omega)$ & 0.911 & 1.027 \\
$S(\mathrm{~V} / \mathrm{K})$ & 0.051 & 0.052 \\
$K(\mathrm{~W} / \mathrm{K})$ & 1.030 & 10.062 \\
\hline
\end{tabular}

values are used in the CFD numerical tool in order to simulate the thermal performance. The thermoelectric cooling performance was initially tested with a CFD cooling model of the Peltier device alone. 


\subsection{Thermoelectric material options}

Thermoelectric Cooler (TEC) region of the numerical model is a function of the mean temperature of the device. Two TEC modules were considered in the analysis; a commercially available Bismuth Telluride (BiTe) module and a custom hybrid unit. The parameters for each device were extracted from the data sheet in order to define the linear equations that describe their behaviour at room temperature [15].

As this is a linear approximation (interpolated between the temperatures) a further complication arises when modelling the system at elevated temperatures [16]. The thermal conductance, electrical resistivity and Seebeck coefficient for the thermoelectric modules vary with temperature, each material having different characteristics. It is necessary to have a mathematical model or measurements of material characteristics at the elevated properties to determine or predict the cooling performance of the TECs. The thermal conductivity $K$ of a material consists of an electrical component and a lattice component. Its given by

$$
\kappa=\kappa_{E}+\kappa_{L}
$$

The electrical component $\kappa_{E}$ is related to the electrical resistivity and can be calculated by Wiedmann-Franz law, $\kappa_{E}=L T / \rho$ where $L$ is the Lorenz number. The $\kappa_{L}$ value was determined using the $\kappa$ values at room temperature and the $\kappa_{E}$ values. This approach results in an error at high temperatures. However, flash thermal diffusivity measurements have given way to better thermal conductivity measurements at higher temperatures. These data are currently available for a range of thermoelectric materials, however, these data are not entirely useful in the CFD calculations. Nevertheless, with the use of a curve fitting technique to match these points closely, it is possible to generate input data for the CFD simulation. A second order relationship is used in this instance that will improve the predictions, as shown later.

\subsection{Test to characterize TEC modules}

Tests were carried out to characterize the TEC performance. The heat transfer properties were measured at different hot side temperatures, viz: 160 ${ }^{\circ} \mathrm{C}, 180{ }^{\circ} \mathrm{C}$ and $225^{\circ} \mathrm{C}$. The modules are fitted into an oven test rig, this comprises a copper heatsink and a vacuum flask. Within the vacuum flask is an aluminium mass (slug), surrounded by mineral wool insulation. The thermoelectric module is clamped between the heatsink and the aluminium mass. Foam insulation is used to fill the gap around the module. Thermocouples 
are located close to the hot and cold side of the TEC within the slug and the heatsink. The module is powered using a bench power supply at a fixed current. The system is placed into a laboratory oven, and allowed to reach steady state. A digital multimeter is used to record the voltage supplied to the TEC. In order to estimate the performance of the TEC modules it is necessary to calculate the thermal performance of the insulation around the slug. By measuring the rate at which the slug heats, it is possible to calculate the effective insulation value for the system, and thus the heat being pumped by the TEC to maintain steady state can be found:

$$
Q_{C} \propto k \times\left(T_{\text {environment }}-T_{\text {slug }}\right)
$$

The heat flow into the slug is the sum of the conduction and radiation into the system. The conduction is function of the difference in the internal and external temperatures however the radiation is a function of the absolute temperatures of the two surfaces. The effect of this is that, at elevated temperatures, the effective conductivity of the system is increased and heat flow into the system increases for a given temperature differential. In order to estimate this, the flask was tested in isolation. The heatsink was removed, and a $30 \mathrm{~mm}$ layer of silicone foam was used to insulate the top of the flask. The system was placed in the oven at $160{ }^{\circ} \mathrm{C}, 180{ }^{\circ} \mathrm{C}$ and $225{ }^{\circ} \mathrm{C}$ and the time to heat the slug observed Figure 3 shows the temperature results of the TEC module using a linear interpolation technique. It shows that the predicted linear values are coherent and are able to predict the TEC performance satisfactorily.

Figure 4 shows the temperature results of the TEC module using the proposed curve fitting technique. It shows that the predicted linear values are coherent and are able to predict the TEC performance more accurately than linear interpolation. The thermal conductivity properties are plotted against temperature for the Peltier device. The relationship can be expressed in a second order curve fitting in the following form.

$$
\kappa=a T^{2}+b T+c
$$

The model co-efficients for $a, b$ and $c$ are found in table 2 . In the initial computational model, a linear characteristic assumption has been made in order to predict Peltier performance at elevated temperature. Figure 5 and Figure 6 show the predicted behaviour of Pertier cooling performance and the measurements at two different temperatures. This behaviour at el- 

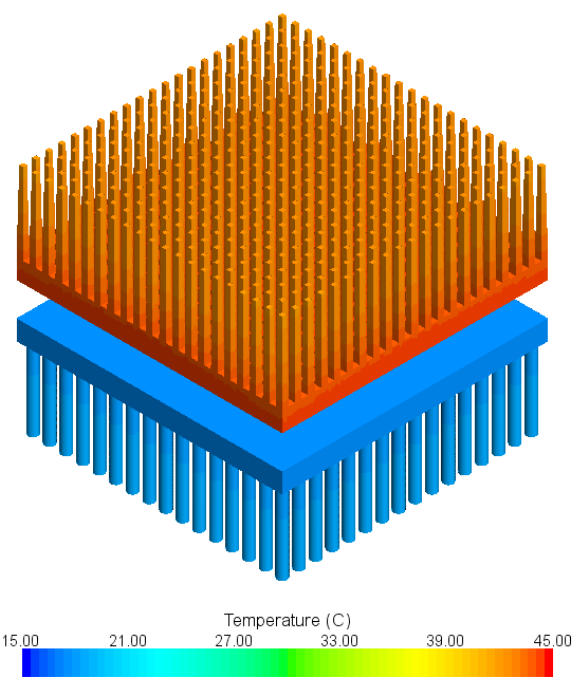

Figure 3: Thermoelectric cooling in the TEC using liner interpolation (Hot side on top)
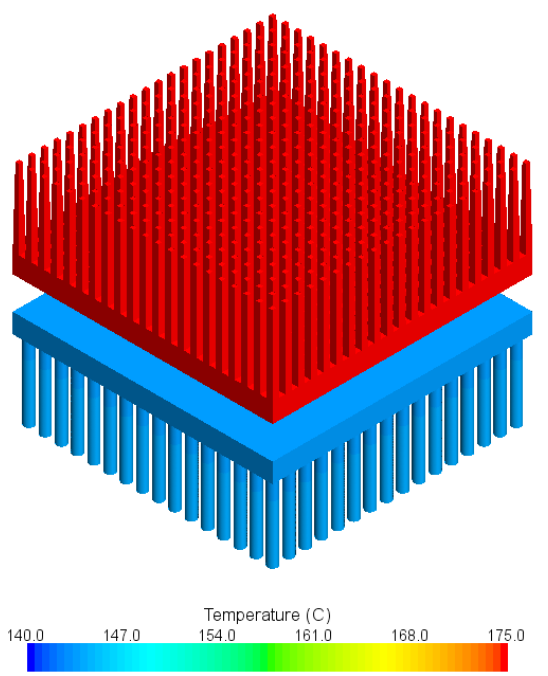

Figure 4: Thermoelectric cooling in the TEC using curve fitting technique (Hot side on top) 
Measurements

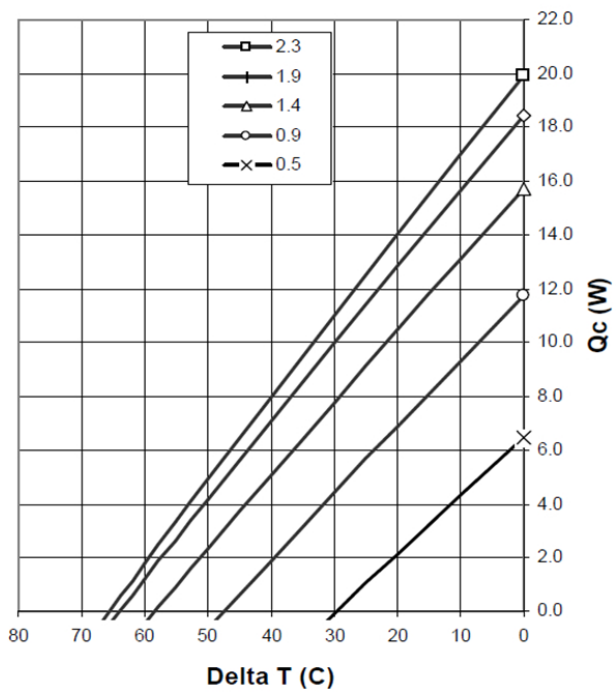

inear interpolation

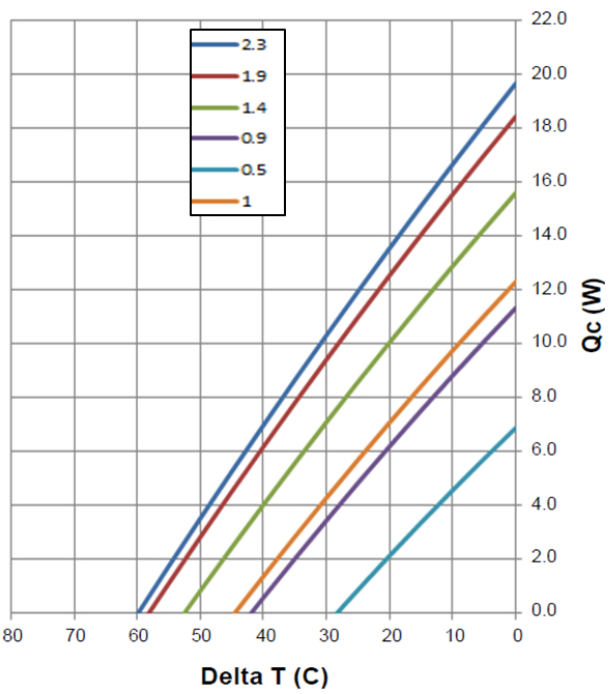

Figure 5: Voltage characteristics for cold side temperature of $25^{\circ} \mathrm{C}$
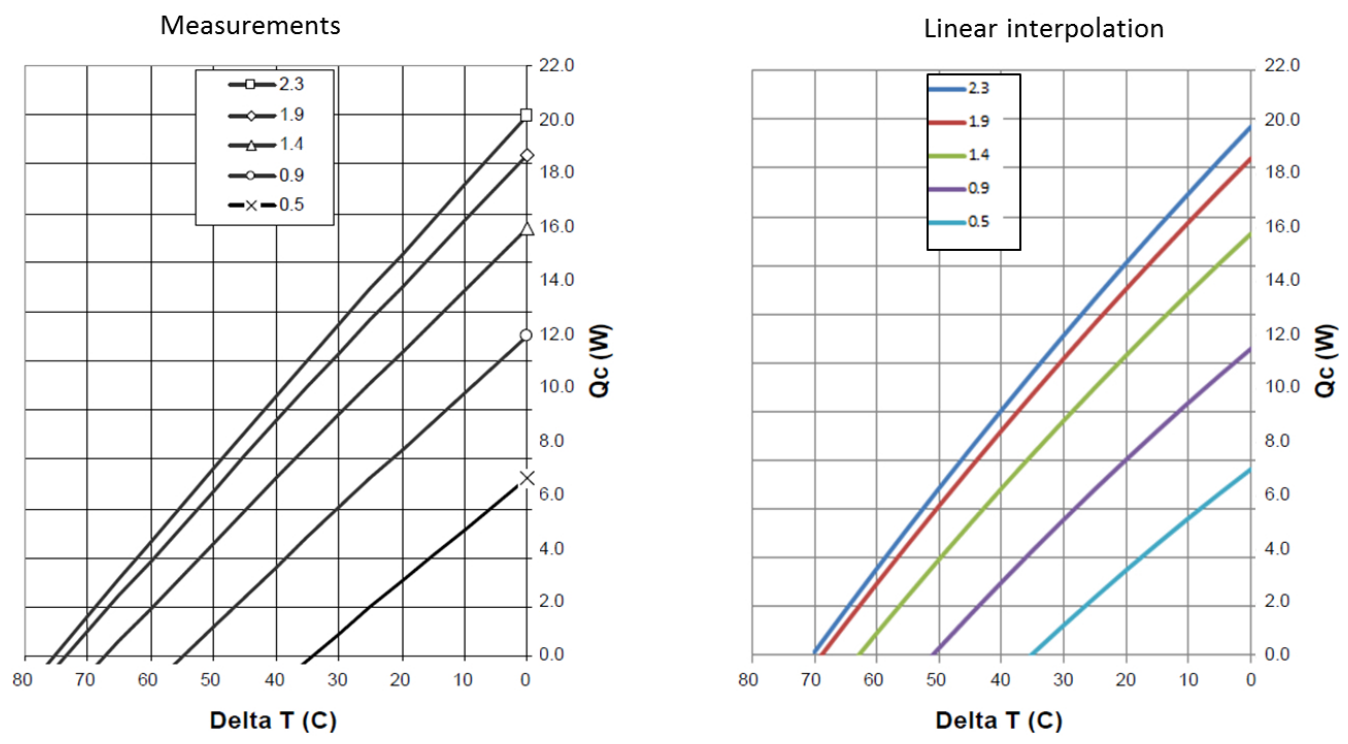

Figure 6: Voltage characteristics for cold side temperature of $50^{\circ} \mathrm{C}$ 
Table 2: Model co-efficiencts for $\kappa$

\begin{tabular}{ll}
\hline \hline Coefficient & Value \\
\hline$a$ & $5.99579472 \mathrm{E}-08$ \\
$b$ & $-4.11897253 \mathrm{E}-05$ \\
$c$ & $7.45107464 \mathrm{E}-03$ \\
\hline
\end{tabular}

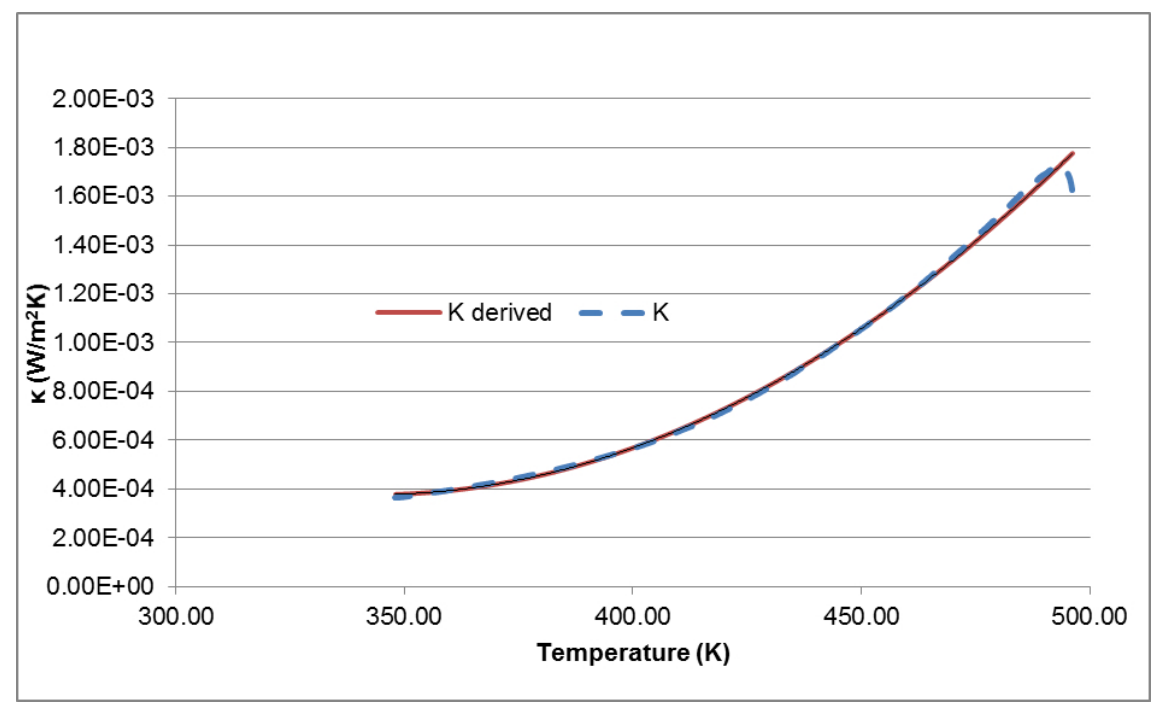

Figure 7: Curve fitting of $\kappa$ behaviour due to change in temperature

evated temperatures are not linearly related. Hence, a more appropriate path would be to determine the shape of the performance and use the same in the numerical code. As such, Figure 7 shows a fitted $\kappa$ value changes due to change in temperature. It can be seen that this is a a very close fit to experimental results. The same is analogous with change in Siebeck co-efficient and resistance. After successful analysis of the Peltier device, the same analytical techniques was used with a model of an actual downhole measuring tool. The prediction technique described above can be used for cooling performance analysis of such tools.

The tool modelled used for the purposes of the study was supplied by Avalon Sciences Ltd. It comprises a steel pressure barrel which houses the geophones, a mechanism to operate an arm which clamps the tool to the wall of the borehole and a module containing the digital electronics which perform the signal processing function. These electronics are housed within 


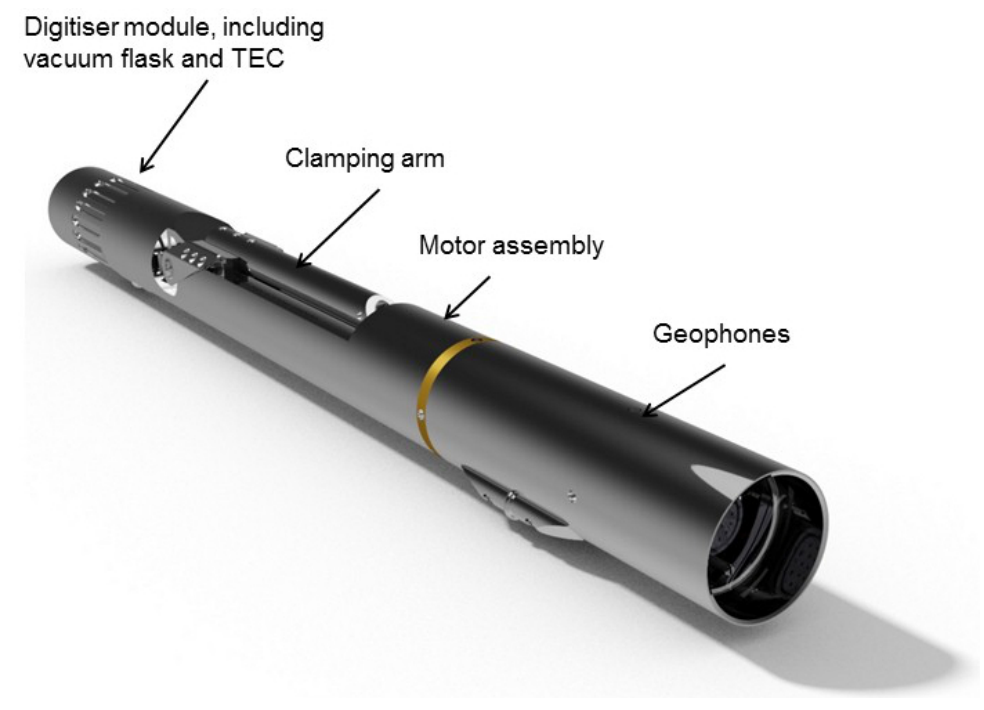

Figure 8: A picture of the downhole measuring tool

a vacuum insulated vessel. Active cooling is provided by a Thermoelectric Cooler (TEC) module similar to one that has been modelled above[25]. The numerical model is described in the chapters to follow.

\section{Numerical model}

The numerical model is a three dimensional representation of the downhole tool similar to one that is commercially manufactured. To expedite the simulation, the regions adjacent to the digital electronics module are excluded from the model as there is no active components in this region and thus have no impact on the cooling of the electronics. To fully resolve all of the the electronic components housed within the module would incur a high computational cost to accurately resolve the geometry and thus a simplified representation of the printed circuit board is used.This simplification does not hinder the performance analysis of heat transfer [19].

The model takes advantage of the symmetry of the tool; only one half of the system is modelled, cut down the central axis of symmetry. Planar symmetry conditions are applied to the cut faces. The external region of the model, representing the well fluid, has a fixed temperature boundary condition on the far face, representing the large thermal capacity of the 
borehole fluid. The fluid is modelled in the laminar regime, with convection driven by gravity in the direction that the tool is oriented in the well. The well fluid is modelled as water.

The starting point of any detailed description of a flows is the conservation equations, namely those describing the conservation of mass and momentum. The derivation of these can be found in many text books on fluid mechanics. Here, for convenience, Cartesian tensor notation is used, where repeated indices, with the exception of Greek symbols imply summation. The conservation of mass can be written as

$$
\frac{\partial \rho}{\partial t}+\nabla \cdot(\rho U)=0
$$

and conservation of momentum yields

$$
\frac{\partial(\rho U)}{\partial t}=-\nabla p+\nabla \tau+S_{M}
$$

where $\tau$ is the stress and $S_{M}$ a body force. Other notations are $\rho$, density, $\nabla$, the partial derivative, $U$, velocity vector with pressure and time denoted by $p$ and $t$, respectively. The fluid is assumed to be Newtonian, hence the stress is supposed to be proportional to the rate of strain. When equation for the stress in the stress tensor form is substituted in (27) and the NavierStokes equations are obtained; These equations are too complex to be solved analytically for the majority of cases and only a limited number of solutions are possible for very simple geometries where the flow is laminar. In the present case laminar flow has been assumed,as there is hardly any turbulence in the well fluid. An additional equation is employed to model heat transfer. This remains separate for the liquid and solid phases. The energy equation for the fluid domain is written as

$$
\frac{\partial\left(\rho h_{\mathrm{tot})}\right.}{\partial t}-\frac{\partial p}{\partial t}+\nabla\left(\rho U h_{\mathrm{tot}}\right)=\nabla(\lambda \nabla T)+\nabla(U \tau)+U S_{M}+S_{E},
$$

and the conservation of energy in the solid domain is given by

$$
\frac{\partial(\rho h)}{\partial t}-\frac{\partial p}{\partial t}+\nabla\left(\rho U_{S} h\right)=\nabla(\lambda \nabla T)+S_{E},
$$

This is the only equation solved in the solid domain. Here $h$ denotes enthalpy and $S$ source terms with subscripts $M$ and $S$ for momentum and energy, respectively. $\lambda$ is the thermal conductivity of the solid. 


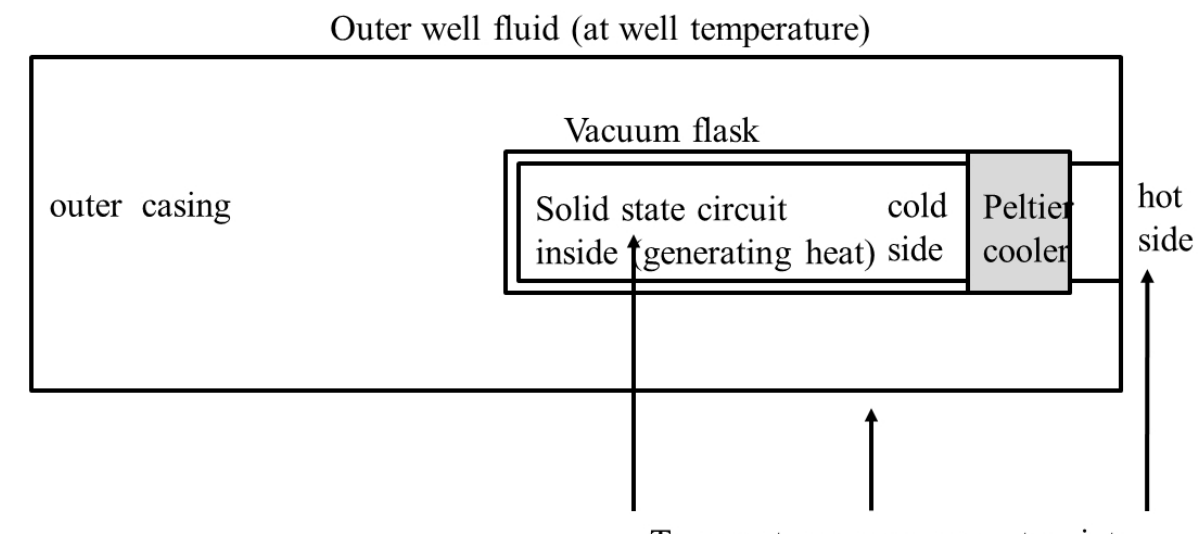

Temperature measurement points

Figure 9: Boundaries and temperature tapping points

\subsection{Problem in Modelling Space}

The mathematical model above can be used in a simulation tool if the temperature and heat flux to be solved for the region. The values defined above can be used to obtain the Seeback coefficient, conductance and resistance as a function of temperature [13], [25]. The values of , (derived using equations 28 and 29 above) and R (obtained from the manufacturer data) were used in a commercially available CFD package Star CCM+ to provide heat flux boundary conditions for the thermoelectric cooling model (TEC). Temperature boundary conditions were set for the hot side of the tool. The above system of six equations would be solved to obtain the heat flux and the temperature field.

A simplified version of the boundaries defined is given in figure9, along with the temperature tapping points. Figure 10 shows the computational mesh with boundary conditions.

The solid regions of the tool were modelled with appropriate material properties, sourced from the manufacturers data sheet. The vacuum region of the flask was modelled as a gas with a low conductivity $\left(1 \times 10^{-6} \mathrm{Wm}^{-1} \mathrm{~K}^{-1}\right)$. Surface to surface radiation was modelled, with the air in the spaces using the participating media model. To expedite the simulation, the whole model was initialised at the borehole temperature, and the electronic packaging region allowed to cool under the action of the TEC. Given that the tools spend many hours, if not months, in well conditions, a steady state model was run 


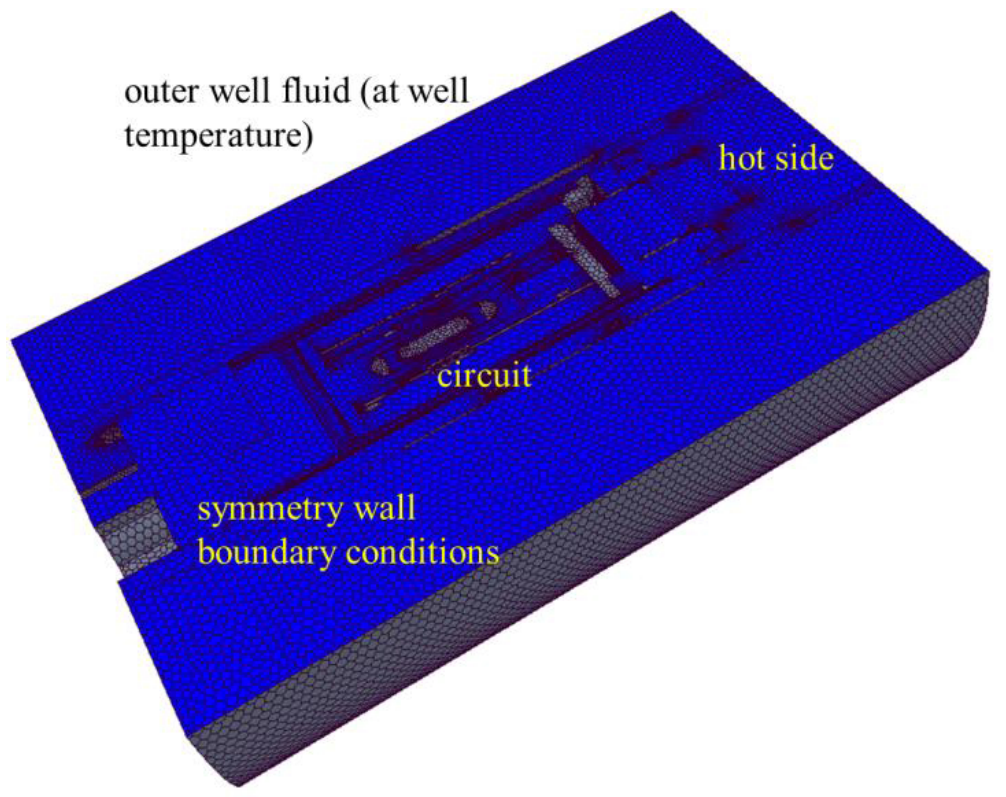

Figure 10: Basic configuration of the tool showing computational mesh

requiring around 4000 iterations to converge to a solution.

\section{Experiments}

Initial validation of the TECs were done using a linear interpolation and a curve fitting technique. Two test modules were considered in the analysis, one Bismuth Telluride (HT2) and the other custom hybrid bi-Te doped with lead (TESH127). In order to derive the linear equations for resistance and Seebeck co-efficients, data from the manufacturers were used. These values were verified with in house testing of the data. The so developed TEC model was validated in the tested temperature range. At elevated temperatures however, the performance could vary as the thermal conductance, electrical resistivity and Seebeck coefficient for the thermoelectric modules vary with temperature, each material having differing characteristics. In order to derive the linear equations that define the performance of the TECs at elevated temperature it is necessary to re-evaluate the manufacturer data values for these temperatures. In the case of the Laird module, it is possible to use the manufacturers analytical design tool, Aztec (Scillasoft, 2014) to find these 
values at the system temperature. When such data are not available, an alternative approach has to be followed. The resistance of the unit at a range of mean temperatures was measured in a laboratory oven and a digital multi meter to record voltage drop across the unit at a fixed current. From these data, a linear equation for the resistance could be derived. In the absence of direct experimental data and extrapolation technique based on measurements and curve fitting was used to find values of zT. [7, 9] plots values for zT at temperature for a range of materials. If the composition of the module was known, values could be estimated from these curves. In the absence of these values, the data from the experimental oven testing was used to approximate zT with temperature. A copper heat sink was used to dissipate heat from the hot side, and the cold side was fixed to an insulated mass. Thermocouples were used to measure the hot and cold side temperatures.

To verify the effectiveness of the geophonic tool, a separate computational analysis was carried out to assess the effectiveness of different materials for heat sinks and heat sink arrangements. Experimental testing has shown a temperature differential around $10{ }^{\circ} \mathrm{C}$ between the hot side of the TEC module and the environment in the tool. Increasing the heat flux in the heat sink should reduce this bottleneck and increase thermal performance. A CFD study was carried out to simulate the heat flow inside the tool with a Colisbo heat sink and a Nibron heat sink, respectively. The physical element was modelled using STAR CCM+. The well fluid was represented by water surrounding the heat sink with $150{ }^{\circ} \mathrm{C}$ convection boundaries with gravity enabled. A heat source of $15 \mathrm{~W}$ was applied to the TEC face of the heatsink. In a simplistic representation (assuming perfect lagging) the other faces of the heat sink were modelled as walls with zero heat flux. Colsibro and Nibron have wall heat conductivities of $188 \mathrm{~W} / \mathrm{mK}$ and $77 \mathrm{~W} / \mathrm{mK}$, respectively. The effect of the two materials are discussed in the results section.

\section{Results}

The purpose of the study was to investigate the feasibility of using the CFD simulation to virtually prototype thermoelectric cooling used in the thermal management of downhole tools, using the information in the data sheet and a simple experimental techniques to characterise the units at elevated temperature. Preliminary CFD studies were carried out to analyse the effect of the heat sink material [33]. The boundary heat fluxes calculated are 


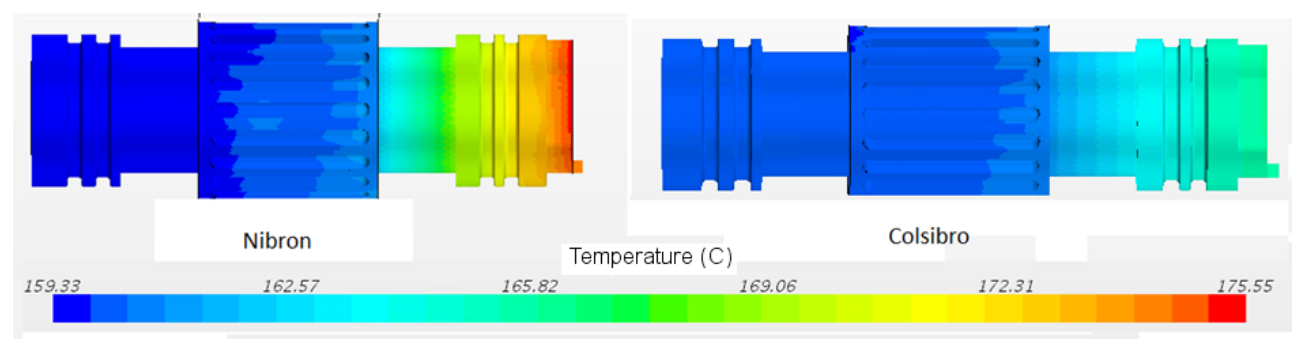

Figure 11: Temperature plots of solid Nibron and Colsibro, hot side to the right of the tool

shown in table 3. It shows the maximum and minimum temperature shown on each test with the heat flux from the tool to the well fluid.

Table 3: Calculated boundary heat fluxes at heat sink

\begin{tabular}{llll}
\hline \hline Test & Heat Flux (W) & $\mathrm{T}_{\min }$ & $T_{\max }$ \\
\hline Colsiblo & -14.91 & 159.78 & 166.10 \\
Nibron & -14.90 & 159.31 & 175.55 \\
\hline
\end{tabular}

The minimum temperature is consistent around $159{ }^{\circ} \mathrm{C}$ and there is a $10{ }^{\circ} \mathrm{C}$ difference in the maximum temperature. Analysis of the temperature profile in the model shows that the greatest temperature differential occurs at the region closest to the TEC. Obviously, the geometry used in this simulation does not show the Nibron locking collar or other hardware which will contribute to the heat path in the tool, but it does provide a comparative value. The full heat sink model shows a lower temperature at the TEC $\left(164.11{ }^{\circ} \mathrm{C}\right)$, but the heat distribution is greater. Figure 11 show the difference in temperature distribution depending on the conductivity. Based on this highly simplified CFD model, changing the heatsink to a material of greater conductivity may help reduced the hot side temperature of the TEC in the GSR tool.

The above analysis proves the importance of thermal conductance of the heat sink of the tool. The discussion below is based on the analysis of the whole tool with a Nibron heat sink.

It has been shown that the first-order linear approximation method provides a steady-state solution that is comparable with experimental data, where resistance of the unit at elevated temperatures can be found from a 
laboratory experiment and the values for $Q_{\max }$ and $\Delta T_{\max }$ can be estimated based on values reported in the literature. However, a curve fitting technique as described earlier provides a closer thermal characteristic representation.

Table 4: Mean temperatures and cooling effects observed in simulations and testing

\begin{tabular}{lllll}
\hline \hline & $\begin{array}{l}\text { Fluid Temp. } \\
\left({ }^{\circ} \mathrm{C}\right)\end{array}$ & $\begin{array}{l}\text { Flask Temp. } \\
\left({ }^{\circ} \mathrm{C}\right)\end{array}$ & $\begin{array}{l}\text { Cooling } \\
\left({ }^{\circ} \mathrm{C}\right)\end{array}$ & $\begin{array}{l}\text { Voltage } \\
(\mathrm{V})\end{array}$ \\
\hline Experiment HT2 & 161.06 & 134.82 & 26.20 & 12.5 \\
CFD HT2 & 160.00 & 133.77 & 26.20 & 11.6 \\
Experiment TESH 127 & 160.37 & 127.57 & 32.80 & 16.5 \\
CFD TESH 127 & 160.00 & 126.40 & 33.60 & 20.6 \\
\hline
\end{tabular}

Figure 12 shows the temperature results of the TEC module using a linear interpolation technique. It shows that the predicted linear values are coherent and are able to predict the tool performance satisfactorily. Moreover it shows the same behaviour shown by the Peltier device alone. The agreement is not ideal, and according to previous studies, the co-efficient of performance at elevated temperatures cannot be accurately predicted based on room temperature results. This is due to the fact that the $\kappa_{E}$ value showing a non linear relationship with the temperature. As explained before, change in $\kappa$ results in a change in the coefficient of performance, hence, the resultant cooling effect. However, this non linear behaviour is so evident at higher temperatures around $700{ }^{\circ} \mathrm{C}$. The agreement around $200{ }^{\circ} \mathrm{C}$ is better than the agreement at such high temperatures. Nevertheless, if the temperature performance is predicted accurately, the results of the numerical model will be more accurate, hence, the attempt to model with a second order relationship.

Figure 13 shows the temperature results of the downhole tool using the proposed curve fitting technique. It shows that the predicted linear values are coherent and are able to predict the performance more accurately than linear interpolation.

\section{Conclusions}

The aims of the present work were to evaluate the performance of the downhole tool with two types of TEC cooling modules and then use the model as a base model in evaluating performance of similar tools. The numerical 


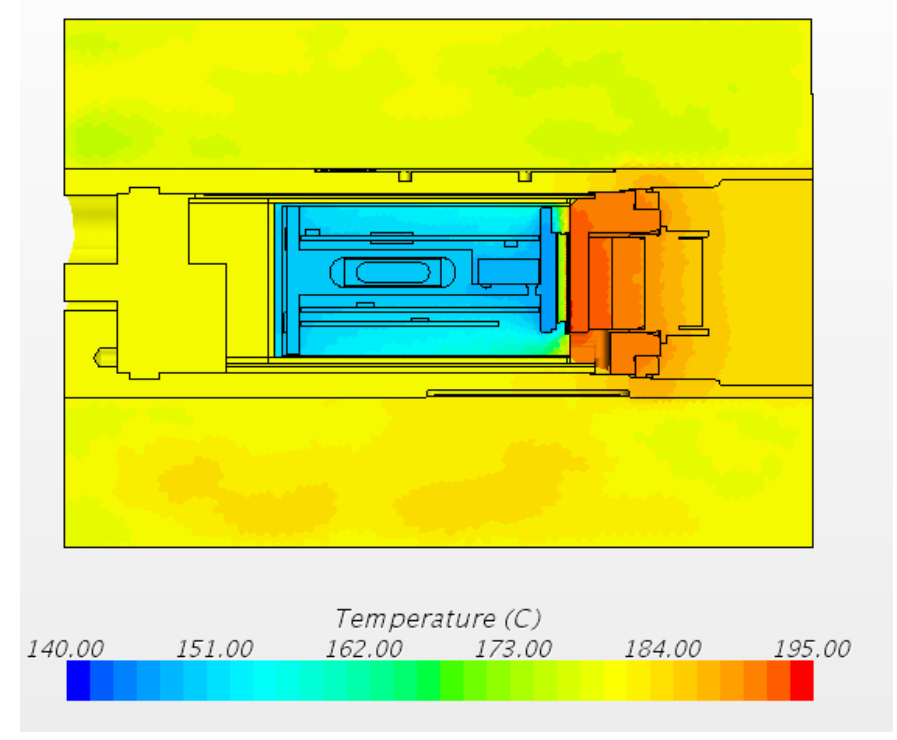

Figure 12: Thermoelectric cooling in the downhole tool using linear interpolation (inside enclosure in blue and high temperatures in red)

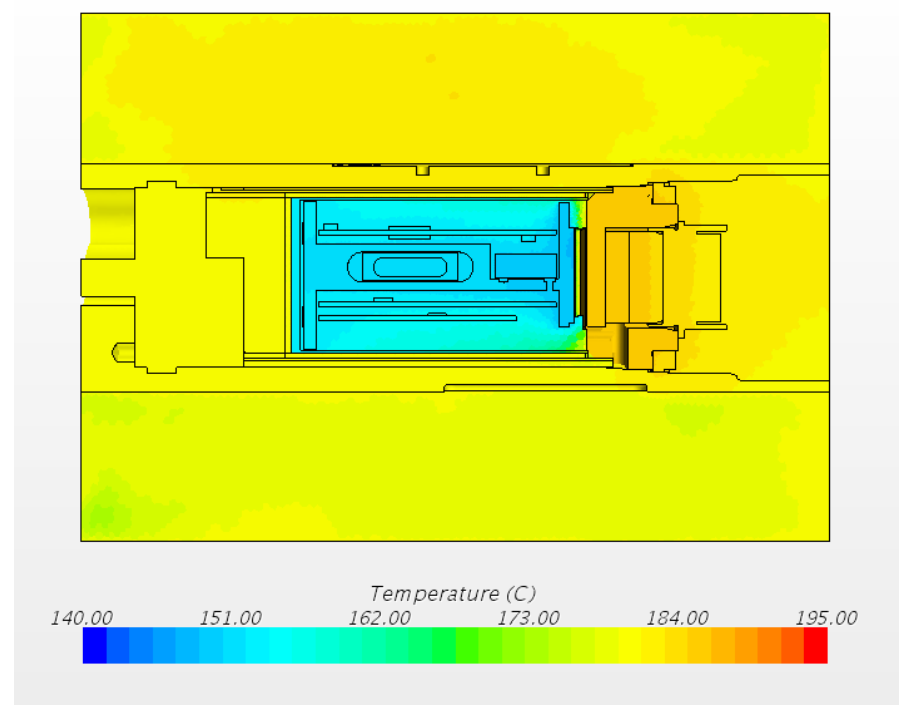

Figure 13: Thermoelectric cooling in the downhole tool using curve fitting technique at elevated temperature (inside enclosure in blue and high temperatures in red) 
model was able to show the difference in performance of the two modules. The temperatures recorded in the electronic region of the module shows good agreement with the experimental values.

Table 4 shows the fluid and flask temperatures and the cooling effect for HT2 and TESH 127, both for experiments and CFD simulations. The temperature drop predicted with the linear model for the TESH 127 Peltier device alone is $50.0 \mathrm{~K}$ is for the linear model and $33.0 \mathrm{~K}$ for the second order model. These values translate into the tooling device as $26.2 \mathrm{~K}$ and $33.6 \mathrm{~K}$, respectively. The agreement with measured values of the linear model and the second order model show the difference between the two techniques. The second order model shows excellent agreement at this temperature range. This shows that the second order model used here is a suitable method for prediction of thermoelectric cooling in this range. The accuracy of the model at higher temperatures in the range of $700{ }^{\circ} \mathrm{C}$ where it is of significance for automotive applications such as thermoelectric heat recovery has to be investigated separately. The second order method however, should be able to predict accurate enough values even at such high temperatures. However, the temperature behaviour of the thermoelectric material cannot be generalized using simple equations. In the present work only the operating region has been tested. Further modelling work in other regions, for example in automotive heat recovery applications $[1,4]$ using thermoelectric devices, have to be done in order to validate the model that has been used here. Those models have to be tested with experimental data. Such modelling is out of the scope of present work, but the technique may be useful at least as an initial model.

With the simulation results agreeing with experimental validation, the present work has opened an avenue for accurate prediction of downhole tool thermal performance. It is possible to design optimized tools with better thermal characteristics with the aid of CFD modelling. This should reduce significant costs in tool design. With more advanced prediction models manufacturers will benefit further without having to validate their designs with prior extensive testing. The modelling process will reduce some of the time consuming and very costly testing procedures.

With the aid of the present work, an existing downhole tool was introduced to the market by Innovate UK and the tool demonstrated significant improvements in on field applications. The experimentation life has been improved of this tool that shows the effectiveness of the improved design. The modelling methodology can be used in similar tools alike. The target 10 
K improvement of tool performance has been achieved with the aid of Peltier cooling and thermal modelling based tool design optimisation. Two variants of the present tool have been modelled and have shown improvements in heat transfer in modelling. They are expected to show similar characteristics to the present tool in operation in the field. The technique is theoretically scalable.

\section{Acknowledgments}

The authors would like to thank Avalon Sciences Ltd for the continual support for the project given as the industry partner. The research work was funded by the knowledge transfer partnership grant No KTP009448 of Innovate UK. The authors also want to mention the contributions in this area by CD-Adapco, especially Rubens Bons of Siemens [3]

[1] P. Aranguren, D. Astrain, A. Rodrguez, and A. Martnez. Net thermoelectric power generation improvement through heat transfer optimization. Applied Thermal Engineering, 120:496-505, 2017.

[2] Shengqiang Bai, Hongliang Lu, Ting Wu, Xianglin Yin, Xun Shi, and Lidong Chen. Numerical and experimental analysis for exhaust heat exchangers in automobile thermoelectric generators. Case Studies in Thermal Engineering, 4:99-112, 2014.

[3] Ruben Bons. Efficiently simulating thermoelectric devices and heat pipes. Unpublished work, 2014.

[4] Murat Emre Demir and Ibrahim Dincer. Performance assessment of a thermoelectric generator applied to exhaust waste heat recovery. Applied Thermal Engineering, 120:696-707, 2017.

[5] P. L. Dreike, D. B. Fleetwood, D. M.and King, D. C. Sprauer, and T. E. Zipperian. An overview of high-temperature electronic device technologies and potential applications. IEEE Trans., 17:594-649, 1994.

[6] D. Enescu and E.O. Virjoghe. A review on thermoelectric cooling parameters and performance. Renewable and Sustainable Energy Reviews, 38:903-916, 2014.

[7] Y. Gelbstein, Z. Dashevsky, and M. P.. Dariel. High performance n-type pbte-based materials for thermoelectric applications. Physica, B:196205, 2005. 
[8] R. W. Gissler. Thermal insulation vessel. Halliburton Energy Services Inc., U.S. Patent, 6(220,346), 2001.

[9] E.R. Hnatek. Practical reliability of electronic equipment and products. Thermal Management, 2002. New York, CRC Press.

[10] Chang Nyung Kim and Jeongho Kim. Numerical examination of the performance of a thermoelectric cooler with peltier heating and cooling. Journal of Electronic Materials, 44(10):3586-3591, 2015.

[11] Woochul Kim, Joshua Zide, Arthur Gossard, Dmitri Klenov, Susanne Stemmer, Ali Shakouri, and Arun Majumdar. Thermal conductivity reduction and thermoelectric figure of merit increase by embedding nanoparticles in crystalline semiconductors. Phys. Lett. Rev., 96, 2006.

[12] V. A. Kutasov, L. N. Lukyanova, and M. Vedernikov. Thermoelectrics Handbook Macro to Nano. CRC, Boca Raton, 2006. Ch. 37.

[13] Z. Luo. A simple method to estimate the physical characteristics of a thermoelectric cooler. Electronics Cooling, 2008.

[14] Yapu Ma, Bofeng Shang, Run Hu, and Xiabing Luo. Thermal management of downhole electronics cooling in oil and gas well logging at high temperature. 17th International Conference on Electronic Packaging Technology, 2016.

[15] M. Magerl, M. Abajian, K. Krause, S. Altrichter, F. Siebenhaar, and M.K. Church. An improved peltier effect-based instrument for critical temperature threshold measurement in cold- and heat-induced urticaria. Journal of the European Academy of Dermatology and Venereology, 29(10):2043-2045, 2015.

[16] Gianluca A Mannella. Peltier cells as temperature control elements: Experimental characterization and modeling. Applied Energy, 63:234245, 2014. ISSN: 1359-4311.

[17] A. Martinez, D. Astrain, A. Rodriguez, and P. Aranguren. Advanced computational model for peltier effect based refrigerators. Applied Thermal Engineering, 95:339-347, 2016. 
[18] F.P McCluskey, R. Grzybowski, and T. Podlesak. High Temperature Electronics. CRC, New York, 1997.

[19] Jeffrey Snyderb Min Chena. Analytical and numerical parameter extraction for compact modeling of thermoelectric coolers. International Journal of Heat and Mass Transfer, 60:689 - 699, 2013.

[20] Randy Normann and David Glowka. Development of a geothermal well inspection camera with active co2 cooling. Geothermal Resources Council Transactions, 40, 2016.

[21] Ronnie Andersson Olle Hgblom. A simulation framework for prediction of thermoelectric generator system performance. Applied Energy, 180:472-482, 2016.

[22] S. Owens and B. Bouldin. Downhole system for extending the life span of electronic components. PES, Inc., U.S. Patent, 5(547,028), 1996.

[23] R.A. Parrot, H. Song, and K. C. Chen. Cooling system for downhole tools. U.S. Patent, 6(336,408), 2002.

[24] H. Scherrer and S. Scherrer. Thermoelectrics Handbook Macro to Nano. CRC, Boca Raton, 2006. Ch. 27.

[25] Bai Shengqiang, Lu Hongliang, Wu Ting, Yin Xianglin, Shi Xun, and Chen Lidong. Numerical and experimental analysis for exhaust heat exchangers in automobile thermoelectric generaters. Case studies in Thermal Engineering, 4:99-112, 2014.

[26] A. Sinha and Y.K. Joshi. Downhole electronics cooling using a thermoelectric device and heat exchanger arrangement. , Jounal of Electronic Packaging, 133(4):041005-1-041005-12, 2012.

[27] D.Y. Sootsman, J.R.and Chung and M.G. Kanatzidis. New and old concepts in thermoelectric materials. Angewandte Chemie (International ed.in English), 48:8616-8639, 2009.

[28] S. Soprania, J.H.K. Haertela, B.S. Lazarovb, O. Sigmund, and K. Engelbrecht. A design approach for integrating thermoelectric devices using topology optimization. Applied Energy, 176:49-64, 2016. 
[29] Zhiting Tian, Sangyeop Lee, and Gang Chen. A comprehensive review of heat transfer in thermoelectric materials and devices. Ann. Rev. Heat Transfer, 17:425-483, 2014.

[30] R. K. Traeger and P. C. Lysne. High temperature electronics application in well logging. IEEE Trans. Nucl. Sci., 35:852-854, 1988.

[31] W. E. Turner, R. Seppa, W. E. Turner, and A. J. Sallwasser. Cooled electrical system for use downhole. APS Technology, Inc., U.S. Patent, 6(134,892), 2000.

[32] Geoff Watson and Gustavo Castro. High temperature electronics pose design and reliability challenges. Analog Dialogue, 46, 2012.

[33] Rohitha Weerasinghe and Thomas Hughes. Numerical and experimental investigation of thermoelectric cooling in down-hole measuring tools; a case study. Case Studies in Thermal Engineering, pages -, 2017.

[34] M. R. Werner and W. R. Fahrner. Review on materials, microsensors, systems and devices for high-temperature and harsh-environment applications. IEEE Trans, 48:249-257, 2001.

[35] D. Zhao and G. Tan. A review of thermoelectric cooling: Materials, modeling and applications. Applied Thermal Engineering, 66:15-24, 2014. No 1-2. 